\title{
ОПРЕДЕЛЕНИЕ КОНСТАНТ ОБРАЗОВАНИЯ Н-СВЯЗИ МЕЖДУ ФЕНОЛАМИ И СЛОЖНЫМИ ЭФИРАМИ ПО ДАННЫМ РАВНОВЕСИЯ ЖИДКОСТЬ-ЖИДКОСТЬ
}

В [1] авторами предложен метод для предсказания коэффициентов распределения протонодонорного вещества А между протоноакцепторным растворителем В и водой по параметрам растворимости и константе образования Н-связи между А и В в неполярном растворителе. При проверке метода на примере распределения одноатомных фенолов между алифатическими простыми эфирами и водой найдено удовлетворительное согласие теории и эксперимента. В настоящей работе сделана попытка использовать теоретические соображения, изложенные в [1], для расчета констант образования Н-связи между фенолами и сложными эфирами по данным межфазового распределения.

\section{Связь $\mathrm{K}_{\mathrm{AB}}$ с коэффициентом распределения}

Из уравнения (4) в ['] после несложного преобразования получим для константы образования Н-связи между фенолом и протоноакцепторным растворителем $\left(\mathrm{K}_{\mathrm{AB}} \equiv \mathrm{a}_{\mathrm{AB}(\mathrm{B})} / \mathrm{a}_{\mathrm{A}(\mathrm{B})} \cdot \mathrm{a}_{\mathrm{B}(\mathrm{B})}\right)$

$$
\mathrm{K}_{\mathrm{AB}}=\left(\mathrm{P}_{\mathrm{A}} / \mathrm{K}_{\mathrm{A}}-1\right) \gamma_{\mathrm{AB}(\mathrm{B})} / \gamma_{\mathrm{A}(\mathrm{B})},
$$

где $\mathrm{P}_{\mathrm{A}} \equiv\left(\mathrm{x}_{\mathrm{A}(\mathrm{B})}+\mathrm{x}_{\mathrm{AB}(\mathrm{B})}\right) / \mathrm{x}_{\mathrm{A}(\mathrm{B})}-$ суммарный коэффициент распределения фенола А между протоноакцепторным растворителем В и водой, $\mathrm{K}_{\mathrm{A}} \equiv$ $\equiv \mathrm{x}_{\mathbf{A}(\mathrm{B})} / \mathrm{x}_{\mathrm{A}(\mathrm{B})}-$ коэффициент распределения не связанного в комплекс мономера A, х - мольная доля, a - активность, $\gamma$ - коэффициент активности, индексы А, в и Ав относятся, соответственно, к веществам A, В и соединению $\mathrm{AB}$, индексы (В) и (в) - к фазам В и воды.

Как показано в ['], растворы фенолов в протоноакцепторных растворителях при бесконечном разбавлении можно рассматривать как регулярные трехкомпонентные $(\mathrm{A}+\mathrm{AB}$ в $\mathrm{B})$, предполагая, что $\mathrm{V}_{\mathrm{AB}} \cong \mathrm{V}_{\mathrm{A}}+\mathrm{V}_{\mathrm{B}}$ и $\delta_{\mathrm{AB}} \cong \frac{\mathrm{V}_{\mathrm{A}}}{\mathrm{V}_{\mathrm{AB}}} \delta_{\mathrm{A}}+\frac{\mathrm{V}_{\mathrm{B}}}{\mathrm{V}_{\mathrm{AB}}} \delta_{\mathrm{B}}(\mathrm{V}$-- молярный объем, $\delta$ - параметр растворимости).

В этом случае при $\mathrm{x}_{\mathrm{A}(\mathrm{B})} \rightarrow 0$ и $\mathrm{x}_{\mathrm{AB}(\mathrm{B})} \rightarrow 0$

$$
\ln \gamma_{A(B)}=\ln \frac{V_{A}}{V_{B}}+\left(1-\frac{V_{A}}{V_{B}}\right)+\frac{V_{A}}{R T}\left(\delta_{A}-\delta_{B}\right)^{2}
$$

и

$$
\ln \gamma_{\mathrm{AB}(\mathrm{B})}=\ln \frac{\mathrm{V}_{\mathrm{AB}}}{\mathrm{V}_{\mathrm{B}}}+\left(1-\frac{\mathrm{V}_{\mathrm{AB}}}{\mathrm{V}_{\mathrm{B}}}\right)+\frac{\mathrm{V}_{\mathrm{AB}}}{\mathrm{RT}}\left(\delta_{\mathrm{AB}}-\delta_{\mathrm{B}}\right)^{2}
$$


$\mathrm{K}_{\mathrm{A}}$ определяется согласно уравнению (5) в [1], если в качестве параметра растворимости воды использовать эмпирическое значение, учитывающее фактическое отклонение водной фазы от регулярности.

Таким образом, $\mathrm{K}_{\mathrm{AB}}$ можно найти по экспериментальным значениям $\mathrm{P}_{\mathrm{A}}$, если известны параметры растворимости и молярные объемы $\mathrm{A}, \mathrm{B}$ и водной фазы.

\section{Экспериментальная часть и обработка результатов}

Коэффициенты распределения фенолов при бесконечном разбавлении (табл. 1) определялись по методике, описанной в [2]. Температуру в опытах поддерживали $20 \pm 1^{\circ} \mathrm{C}$. Как правило, результаты параллельных опытов не различались больше, чем на $2-3 \%$.

Таблица 1

$\mathbf{P}_{\mathbf{A}}$ при распределении фенолов (мд/мд) между сложными эфирами и водой при $20^{\circ} \mathrm{C}$

\begin{tabular}{|c|c|c|c|c|c|c|}
\hline Сложный эфир & $\begin{array}{c}V_{\mathrm{B}} \\
C \mathrm{M}^{3} / M O \Omega b\end{array}$ & Фенол & $\begin{array}{c}\text { м-Кре- } \\
\text { зол }\end{array}$ & $\begin{array}{c}n \text {-Kpe- } \\
\text { зол }\end{array}$ & $\begin{array}{c}\text { 3,4-Кси- } \\
\text { ленол }\end{array}$ & $\begin{array}{c}\text { 3,5-Кси- } \\
\text { ленол }\end{array}$ \\
\hline н-Бутилацетат & 131,8 & 384 & 1031 & 1034 & 2348 & 2618 \\
\hline н-Амилацетат & 148,9 & 399 & 1075 & 1136 & 2380 & - \\
\hline изо-Амилацетат & 149,2 & 369 & 1018 & 1052 & - & - \\
\hline н-Пропилпропионат & 131,8 & 361 & 989 & 995 & 2165 & 2421 \\
\hline H-Бутилпропионат & 148,7 & 331 & 834 & 829 & 1779 & - \\
\hline изо-Амилпропионат & 165,8 & 303 & 834 & 836 & 1808 & 2052 \\
\hline н-Октилпропионат & 215,5 & 349 & 967 & 980 & 2057 & 2201 \\
\hline Этилбутират & 132,2 & 367 & 988 & 1013 & 2201 & 2527 \\
\hline н-Пропилбутират & 149,1 & 338 & 900 & 903 & 1969 & 2176 \\
\hline н-Бутилбутират & 165,8 & 322 & 881 & 893 & 1987 & 2107 \\
\hline изо-Бутилбутират & 167,3 & 290 & 821 & 817 & 1686 & 1848 \\
\hline изо-Амилбутират & 182,9 & 279 & 741 & 734 & 1624 & 1877 \\
\hline Этилизовалерат & 150,4 & 312 & 818 & 823 & 1819 & 2028 \\
\hline
\end{tabular}

Фенолы перед использованием очищались по методике, описанной в [2]. Сложные эфиры несколько раз промывались насыщенными растворами двууглекислого и хлористого натрия, а затем ректифицировались. Неполярные растворители, использованные для определения параметров растворимости ксиленолов, очищались способами, предложенными в [3].

Параметры растворимости сложных эфиров (табл. 2) рассчитывали по $\left.{ }^{4}\right]$ из теплот испарения при $20^{\circ}$, полученных по уравнению РиделяПланка-Миллера и корреляции Ватсона [5] из критических параметров. $\mathrm{T}_{\mathrm{kp}}$ и $\mathrm{p}_{\mathrm{kp}}$ находили методом Герцога $\left[{ }^{6}\right]$ по парахору и температуре кипения. Парахор рассчитывали по [7]. За исключением бутилформиата, вели-

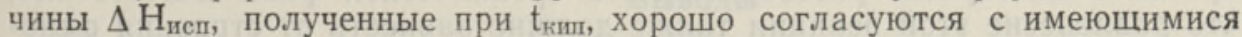
литературными данными (для 21 эфира среднее отклонение $1,70 \%$, максимальные $+2,71$ и $-2,89 \%)$. Это дает право предполагать, что параметры растворимости эфиров определены с точностью в среднем $\pm 0,9 \%$.

Для фенола и крезолов использовали значения $\delta_{\mathrm{A}}$ и $\delta_{\mathrm{B}}$, найденные ранее в $\left[{ }^{2}\right]$. Для определения $\delta_{\mathrm{A}}$ и $\delta_{\text {в }}$ для ксиленолов проводились специальные опыты экстракции этих фенолов растворителями, не образующими с фенолами Н-связи. Результаты расчета $\delta_{\mathrm{A}}$ и $\delta_{\mathrm{B}}$ из экспериментальных данных по методике, изложенной в [2], приводятся в табл. 3.

Из данных табл. 1 явствует, что при любом экстрагенте соотношение значений $\mathrm{P}_{\mathrm{A}}$ для отдельных фенолов почти постоянно: крезолы извлекаются из воды в среднем в 2,7, 3,4-ксиленол - в 5,9 и 3,5 -ксиленол - 
Теплоты испарения $\left(\Delta \mathbf{H}_{\text {Iсп }}, \kappa a л /\right.$ моль $)$ и параметры растворимости $\left(\delta_{\mathrm{B}},\left(\kappa a \Omega / c M^{3}\right)^{0,5}\right)$ при $20^{\circ} \mathrm{C}$ для сложных эфиров $\mathrm{R}-\mathrm{C}$

\begin{tabular}{|c|c|c|c|c|c|c|c|c|}
\hline \multirow{2}{*}{$\mathrm{R}^{\prime}$} & \multicolumn{2}{|c|}{$\mathrm{R}=\mathrm{H}-$} & \multicolumn{2}{|c|}{$\mathrm{R}=\mathrm{CH}_{3}-$} & \multicolumn{2}{|c|}{$\mathrm{R}=\mathrm{C}_{2} \mathrm{H}_{5}-$} & \multicolumn{2}{|c|}{$\mathrm{R}=\mathrm{C}_{3} \mathrm{H}_{7}-$} \\
\hline & $\Delta \mathrm{H}_{\mathrm{Ir} \text { त }}$ & $\delta_{B}$ & $\Delta \mathrm{H}_{\mathrm{u}} \mathrm{c \pi}$ & $\delta_{B}$ & $\Delta \mathrm{H}_{\mathrm{nc \text {II }}}$ & $\delta_{\mathrm{B}}$ & $\Delta \mathrm{H}_{\mathrm{n} \in \mathrm{n}}$ & $\delta_{\mathrm{B}}$ \\
\hline $\begin{array}{l}\mathrm{CH}_{3}- \\
\mathrm{C}_{2} \mathrm{H}_{5}- \\
\mathrm{C}_{3} \mathrm{H}_{7}- \\
\left(\mathrm{CH}_{3}\right)_{2} \mathrm{CH}- \\
\mathrm{C}_{4} \mathrm{H}_{9}- \\
\left(\mathrm{CH}_{3}\right)_{2} \mathrm{C}_{2} \mathrm{H}_{4}- \\
\mathrm{C}_{5} \mathrm{H}_{11}- \\
\left(\mathrm{CH}_{3}\right)_{2} \mathrm{C}_{3} \mathrm{H}_{6}- \\
\mathrm{C}_{8} \mathrm{H}_{17}-\end{array}$ & $\begin{array}{r}6718 \\
7532 \\
8551 \\
8089 \\
9544 \\
9250 \\
10540 \\
10290 \\
13080\end{array}$ & $\begin{array}{l}9,96 \\
9,30 \\
9,04 \\
8,65 \\
8,85 \\
8,64 \\
8,71 \\
8,60 \\
8,50\end{array}$ & $\begin{array}{r}7634 \\
8406 \\
9367 \\
8934 \\
10390 \\
10030 \\
11380 \\
11110 \\
13760\end{array}$ & $\begin{array}{l}9,35 \\
8,94 \\
8,74 \\
8,44 \\
8,63 \\
8,42 \\
8,52 \\
8,40 \\
8,34\end{array}$ & $\begin{array}{r}8500 \\
9284 \\
10290 \\
9776 \\
11250 \\
10930 \\
12170 \\
12000 \\
15320\end{array}$ & $\begin{array}{l}9,07 \\
8,72 \\
8,58 \\
8,28 \\
8,47 \\
8,31 \\
8,35 \\
8,30 \\
8,27\end{array}$ & $\begin{array}{r}9394 \\
10220 \\
11170 \\
10680 \\
12160 \\
11890 \\
13140 \\
12890 \\
15700\end{array}$ & $\begin{array}{l}8,80 \\
8,54 \\
8,42 \\
8,16 \\
8,36 \\
8,22 \\
8,29 \\
8,20 \\
8,22\end{array}$ \\
\hline
\end{tabular}

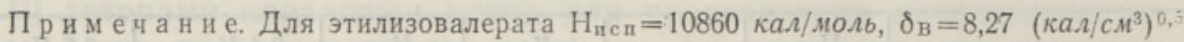

таблица 3

Значения $\delta_{\mathrm{A}}$ и $\delta_{\mathrm{B}}$ для фенолов

\begin{tabular}{|c|c|c|c|}
\hline Фенол & $\begin{array}{c}\text { Молярный } \\
\text { объем при } \\
20^{\circ} \mathrm{C}\left(\mathrm{V}_{\mathrm{A}}\right) \\
\text { см }\end{array}$ & $\begin{array}{c}\delta_{\mathrm{A}}, \\
\left(\kappa a \wedge / \mathrm{CM}^{3}\right)^{0,5}\end{array}$ & $\begin{array}{c}\delta_{\mathrm{B}}, \\
\left(\kappa a \Omega / \mathrm{CM}^{3}\right)^{0,5}\end{array}$ \\
\hline $\begin{array}{l}\text { Фенол } \\
\text { м-Крезол } \\
n \text {-Крезол } \\
3,4 \text {-Ксиленол } \\
3,5 \text {-Кснленол }\end{array}$ & $\begin{array}{r}87,7 \\
104,6 \\
104,5 \\
118,6 \\
120,1\end{array}$ & $\begin{array}{l}12,60 \pm 0,14 \\
12,24 \pm 0,10 \\
12,23 \pm 0,09 \\
11,32 \pm 0,07 \\
11,30 \pm 0,07\end{array}$ & $\begin{array}{r}19,0 \pm 0,21 \\
19,0 \pm 0,15 \\
19,0 \pm 0,14 \\
18,05 \pm 0,11 \\
18,05 \pm 0,11\end{array}$ \\
\hline
\end{tabular}

в 6,6 раза лучше фенола. Такая же закономерность наблюдается для $\mathrm{K}_{\mathrm{A}}$. Обычно значения $\mathrm{P}_{\mathrm{A}}$ и $\mathrm{K}_{\mathrm{A}}$ располагаются в порядке уменьшения длины углеводородных радикалов R и $\mathrm{R}^{\prime}$ в сложном эфире RCOOR', хотя указанная закономерность проявляется менее отчетливо.

\section{Корреляция между $\lg \mathrm{K}_{\mathrm{AB}}$ и структурой $\mathrm{A}$ и $\mathrm{B}$}

Одним из факторов, определяющих межфазовое распределение, является образование Н-связи между распределяемым веществом и протоноакцепторным растворителем в органической фазе. Естественно, что равновесие этой реакции также должно зависеть от строения фенола и сложного эфира, участвующих в реакции комлексообразования. Поэтому в настоящей работе пытались найти зависимость между $\mathrm{K}_{\mathrm{AB}}$, рассчитанной из экспериментальных значений $\mathrm{P}_{\mathrm{A}}$ по уравнению (1), и структурой взаимодействующих веществ.

Действительно, регрессионный анализ данных табл. 4 на ЭВМ показал, что значения $\lg \mathrm{K}_{\mathrm{AB}}$ хорошо коррелируют, с одной стороны, с полярными константами' $\sigma^{*}$ заместителей $\mathrm{R}$ и $\mathrm{R}^{\prime}$ в сложном эфире $\mathrm{RCOOR}$ ( $\sigma^{*}$ взяли из $\left.\left[{ }^{8}\right]\right)$, и, с другой стороны, с константой взаимодействия данного фенола с водой, количественно характеризующейся величиной $\left(\delta_{\mathrm{B}}-\delta_{\mathrm{A}}\right)^{2}$. Путем минимизации дисперсии значений $\lg \mathrm{K}_{\mathrm{AB}}$ нашли коэффициенты b корреляционного уравнения 
таблица 4

$\lg \mathrm{K}_{\mathrm{AB}}$ комплексов фенолов со сложными эфирами при $20^{\circ} \mathrm{C}$, рассчитанные из коэффициентов распределения при бесконечном разбавлении

\begin{tabular}{|c|c|c|c|c|c|}
\hline Сложный эфир & Фенол & м-Крезол & $n$-Крезол & $\begin{array}{c}\text { 3,4-Кси- } \\
\text { ленол }\end{array}$ & $\begin{array}{c}\text { 3,5-Кси- } \\
\text { ленол }\end{array}$ \\
\hline н-Бутилацетат & 1,239 & 1,126 & 1,112 & 1,152 & 1,154 \\
\hline н-Амилацетат & 1,258 & 1,159 & 1,168 & 1,184 & - \\
\hline изо-Амилацетат & 1,247 & 1,160 & 1,165 & - & - \\
\hline н-Пропилпропионат & 1,223 & 1,119 & 1,112 & 1,130 & 1,132 \\
\hline н-Бутилпропионат & 1,187 & 1,059 & 1,041 & 1,066 & - \\
\hline изо-Амилпропионат & 1,164 & 1,079 & 1,071 & 1,109 & 1,120 \\
\hline н-Октилпропионат & 1,177 & 1,104 & 1,094 & 1,149 & 1,135 \\
\hline Этилбутират & 1,237 & 1,132 & 1,128 & 1,145 & 1,161 \\
\hline н-Пропилбутират & 1,207 & 1,106 & 1,092 & 1,123 & 1,123 \\
\hline н-Бутилбутират & 1,180 & 1,091 & 1,087 & 1,137 & 1,118 \\
\hline изо-Бутилбутират & 1,160 & 1,094 & 1,065 & 1,096 & 1,092 \\
\hline изо-Амилбутират & 1,129 & 1,034 & 1,021 & 1,076 & 1,096 \\
\hline Әтилизовалерат & 1,199 & 1,105 & 1,092 & 1,125 & 1,128 \\
\hline
\end{tabular}

$$
\lg \mathrm{K}_{\mathrm{AB}}=\mathrm{b}_{0}+\mathrm{b}_{1} \sigma_{\mathrm{R}}^{*}+\mathrm{b}_{2} \sigma_{\mathrm{R}^{\prime}}^{*}+\mathrm{b}_{3}\left[\mathrm{~b}_{4}-\left(\delta_{\mathrm{B}}-\delta_{\mathrm{A}}\right)^{2}\right]
$$

При уровне достоверности 95\% * они имеют следующие значения: $b_{0}=1,4685( \pm 0,0058), b_{1}=0,6476( \pm 0,0183), b_{2}=1,7194 \quad( \pm 0,0713), b_{3}=$ $=0,01904( \pm 0,00038), b_{4}=40,96$.

Нетрудно убедиться, что при таком выборе входных параметров корреляционного уравнения коэффициент $b_{0}$ представляет собой $\lg \mathrm{K}_{\mathrm{AB}}$ для системы фенол - метилацетат, a $\mathrm{b}_{4}-$ значение $\left(\delta_{\mathrm{B}}-\delta_{\mathrm{A}}\right)^{2}$ для фенола.

Не было обнаружено непосредственной связи между $\lg \mathrm{K}_{\mathrm{AB}}$ и пространственными константами $\left(\mathrm{E}_{8}\right)$ заместителей $\mathrm{R}$ и $\mathrm{R}^{\prime}$. Явно не имеется также корреляции между $\lg \mathrm{K}_{\mathrm{AB}}$ и константами Гаммета заместителей бензольного ядра в молекуле фенола. Последнее обстоятельство, по-видимому, объясняется тем, что склонность фенолов к образованию Н-связи вообще мало зависит от $\boldsymbol{M}$ - и $n$-замещения, не приводящего к пространственным препятствиям.

По нашему мнению, константы образования Н-связи, рассчитанные по уравнению (4), могут использоваться также для предсказания коэффициентов распределения других одноатомных фенолов (за исключением o-замещенных) между сложными эфирами и водой, если для них известны $\delta_{\mathrm{A}}$ и $\delta_{\mathrm{B}}$.

\section{Выводы}

1. Предложен метод расчета констант образования Н-связи между фенолами и сложными эфирами по данным распределения фенолов в двухфазной системе эфир-вода.

2. На основании экспериментальных данных найдено корреляционное уравнение, связывающее константу образования Н-связи с полярными константами заместителей в сложном эфире и константой взаимодействия фенола и воды.

* Стандартная ошибка $\mathrm{s}_{\mathrm{b}}$ соответственно: $\mathrm{s}_{\mathrm{b}_{0}}=0,0228, \quad \mathrm{~s}_{\mathrm{b}_{\mathrm{f}}}=0,0715, \quad \mathrm{~s}_{\mathrm{b}_{7}}=0,2786$, $\mathrm{S}_{\mathrm{b}_{3}}=0,0015$, 
1. Мэлдер Л., Т ам вели ус Х., Изв. АН ЭССР, Хим. Геол., 22, 26 (1973).

2. Мэлдер Л., Т амвели ус Х., Изв. АН ЭССР, Хим. Геол., 21, 204 (1972).

3. В а й с бе егер А., П р оск а уер Э., Рид дик Дж., Т у п с Э., Органические растворители, М., 1958.

4. Hildebrand J. H., Scott R. L., The solubility of nonelectrolytes, 3-rd Ed., Dover, New York, 1964.

5. Р ид Р., Шервуд Т., Свойства газов и жидкостей, Л., 1971.

6. Her zog R., Ind. Engng Chem., 36, 997 (1944).

7. Meissner H. P., Chem. Engng Progr., 45, 149 (1949).

8. Справочник химика, 3, М.-Л., 1964.

Таллинский политехнический институт

Поступила в редакцию 3/XI 1972

\section{MOLDER, H. TAMVELIUS}

FENOOLIDE JA ESTRITE VAHELISE VESINIKSIDEME TASAKAALUKONSTANTIDE ARVUTAMINE VEDELIKU-VEDELIKU TASAKAALUST

Esitatakse meetod fenoolide ja estrite vahelise vesiniksideme tasakaalukonstandi $\left(\mathrm{K}_{\mathrm{AB}}\right)$ arvutamiseks fenoolide jaotuskoefitsientidest estrite ja vee vahel. Antakse korrelatsioonivõrrand, mis seob $\mathrm{K}_{\mathrm{AB}}$ estri struktuuri ning fenooli ja vee vahelise interaktsiooni konstandiga.

\section{MOLDER, H. TAMVELIUS}

\section{CALCULATION OF HYDROGEN-BONDING EQUILIBRIUM CONSTANTS OF PHENOLS TO ESTERS FROM THE LIQUID-LIQUID EQUILIBRIUM}

A method for calculating of $\mathrm{H}$-bonding equilibrium constants $\left(\mathrm{K}_{\mathrm{AB}}\right)$ of some phenols to aliphatic esters from the partition data of phenols between esters and an aqueous solution has been proposed. The constants $K_{A B}$ have been correlated with substituent constants of esters and with interchange energy density for the water-phenol pair by the equation

$$
\lg K_{A B}=b_{0}+b_{1} \sigma_{R}^{*}+b_{2} \sigma_{R^{\prime}}^{*}+b_{3}\left[b_{4}-\left(\delta_{b}-\delta_{A}\right)^{2}\right] \text {, where } \sigma_{R}^{*} \text { and } \sigma_{R^{\prime}}^{*} \text { are Taft's] }
$$

$\sigma^{*}$ values for $R$ and $R^{\prime}$ of the esters $R C O O R^{\prime}, \delta_{A}$ and $\delta_{b}$ - the solubility parameters of phenol and water. The values of constants are: $b_{0}=1.4685( \pm 0.0058), \quad b_{1}=0.6476$ $( \pm 0.0183), b_{2}=1.7194( \pm 0.0713), b_{3}=0.01904( \pm 0.00038), b_{4}=40.96$. 